\title{
A Questão da Agência em Redes Acadêmicas de Pesquisa: Centralidade, Produtividade e Escolha Preferencial
}

\author{
Luciano Rossoni ${ }^{1}$, Edson Ronaldo Guarido Filho ${ }^{2}$ (Universidade Positivo, Brasil) y
} Clóvis L. Machado-da-Silva ${ }^{3}$ (Universidade Positivo e UFPR, Brasil)

\section{Resumo}

No presente artigo, investigamos o papel da agência na construção do conhecimento científico com base em pressupostos do estruturacionismo. Para tanto, a noção de agência foi empiricamente tratada a partir da análise de redes sociais, tendo como indicadores a centralidade, produtividade e escolha preferencial de pesquisadores do campo da pesquisa na área dos estudos organizacionais e da estratégia em organizações no Brasil. Foram considerados 2.332 artigos entre os anos de 1997 e 2005, por meio dos quais analisamos 2.072 pesquisadores. Destacamos o papel de alguns deles, classificados como continuantes, para avaliar mais detalhadamente indicadores estruturais, além da trajetória de sua produção acadêmica. Nossos achados permitem afirmar que não somente a estrutura social apresenta dualidade, condicionando e habilitando a ação, mas também a própria capacidade de agência. Vista correntemente na literatura como potencialmente transformadora, ela também condiciona a ação dos agentes, pois suas capacidades projetivas tendem a ser guiadas por condutas do passado, no sentido de vincular suas ações à trajetória social e intelectual vivida no campo, podendo acarretar maior conformidade e habitualidade na pesquisa. Denominamos esse fenômeno como paradoxo da capacidade de agência, pois a mesma via que habilita a ação também a limita cognitivamente.

Palavras-chave: agência, análise de redes sociais, centralidade, conhecimento científico, escolha preferencial.

\begin{abstract}
In this article, we examine the role of the agency in the construction of scientific knowledge under the assumptions of structurationism. For this purpose, the notion of agency has been empirically treated from the social network analysis, with the indicators being the centrality, productivity and preferential attachment of researchers in the field of organizational studies and strategy in Brazil. We analyzed 2,072 researchers, in that we highlight the role of some of them, classified as continuants, in order to evaluate structural indicators in more detail, in addition to the trajectory of their academic production. Our findings allow us to affirm that it is not only the social structure that shows duality, conditioning and enabling the action, but also the capacity of agency itself. Currently viewed in the literature as a transforming capacity, it also conditions the action of agents because their projective capacities tend to be guided by past conduct in that they link their actions to the social and intellectual trajectory they have experienced in the field, bringing greater conformity and habitualness to research. We have addressed this phenomenon into a cognitive realm and have called it as paradox of agency capability.
\end{abstract}

Keywords: agency, social network analysis, Centrality, scientific knowledge, preferential attachment.

\footnotetext{
${ }^{1}$ Professor do Programa de Mestrado e Doutorado em Administração da Universidade Positivo. E-mail: Irossoni@gmail.com.

${ }^{2}$ Professor do Programa de Mestrado e Doutorado em Administração da Universidade Positivo. E-mail: edson.guarido@gmail.com

${ }^{3}$ Professor Titular do Programa de Mestrado e Doutorado em Administração da Universidade Positivo e Professor Titular do Centro de Pesquisa e Pós-graduação em Administração da Universidade Federal do Paraná (UFPR). E-mail: clms@terra.com.br
} 


\section{I ntrodução}

Toda teoria social pressupõe, por um lado, uma visão de ser humano na tentativa de compreender o fenômeno da ação e, por outro, alguma concepção a respeito da questão da ordem, ou seja, quanto ao modo como padrões e instituições sociais condicionam as ações (Alexander, 1984). Tratam-se dos pressupostos acerca do relacionamento entre agência e estrutura, freqüentemente debatido nos estudos organizacionais (Astley \& Van De Ven, 1983; Reed, 2003; Weaver \& Gioia, 1994).

No estudo da construção do conhecimento científico, considerações ligadas a esse debate também se fazem presentes. Convencionalmente, aspectos estruturais predominam em estudos de redes de cientistas que focam padrões de interação e cooperação ou ainda a influência intelectual por meio de citações recebidas. Nessas situações a questão da agência tende a ficar em segundo plano e naqueles casos em que ela ganha ênfase, a discussão trata mais da avaliação de padrões de ação do que dos mecanismos que os influenciam. Deste modo, em trabalhos orientados para a avaliação do campo científico, predomina a atribuição de maior ênfase na apreciação de características estruturais do que em suas particularidades, como se verifica em Moody (2004), Newman (2001), e Wagner e Leydesdorff (2005).

No presente artigo, adotamos o pressuposto de que o campo científico é um campo social, no qual o conhecimento é construído socialmente, por meio da ação de pesquisadores, produzindo e reproduzindo estruturas que orientam a atividade acadêmica (Machado-da-Silva, Guarido Filho \& Rossoni, 2006). À luz dessa consideração, atribuímos caráter social ao empreendimento científico que, como tal, não está imune à ação humana. Assim, em consonância com essa linha de pensamento, concordamos com DiMaggio (1995, p. 396) no entendimento de que toda

construção teórica é um empreendimento cooperativo entre autores e leitores: a recepção de uma teoria sustenta-se em muito mais do que seu potencial científico; no curto prazo, tendemos a reduzir teorias a slogans [utilizando-as indistintamente]; e no longo prazo, brilhantes analistas podem transformar teorias confusas em obras canônicas [rearticulando-as]. Se o primeiro [...] salienta a necessidade de teóricos exercitarem julgamento e escolha, o segundo sugere a importância do ambiente e do acaso.

Nessa linha de argumentação, discutimos no presente trabalho o papel da agência na construção do conhecimento científico, tomando como referência suas características relacionais e temporais. Nossos argumentos se sustentam sobre o quadro analítico da perspectiva da teoria institucional fundamentado nos pressupostos da teoria da estruturação de Giddens (2003). Com base nisso, objetivamos investigar a capacidade de agência de pesquisadores no campo da 
pesquisa em Estudos Organizacionais e Estratégia em Organizações no Brasil, a partir de aspectos associados às características de produtividade, centralidade e escolha preferencial desses pesquisadores. Para tanto, consideramos como unidade de análise os pesquisadores e seus relacionamentos de co-autoria, avaliados empiricamente por meio de indicadores gerados a partir do método de análise de redes sociais. Os relacionamentos de cooperação entre pesquisadores, por sua vez, foram avaliados a partir da produção conjunta de artigos científicos.

Na primeira parte deste trabalho, realizamos uma revisão do conceito de agência, sob a ótica da perspectiva estruturacionista. Duas abordagens são enfatizadas: a de Giddens (2003), que considera a agência como capacidade transformadora potencial do indivíduo, e a de Emirbayer e Mische (1998), que aprofunda o caráter relacional e temporal da agência. Na sequência, usamos argumentos para apontar que o pesquisador pode ser visto como agente capaz de influenciar o campo científico, por meio de sua capacidade de angariar e cultivar relacionamentos, direcionando e atuando como referência para o trabalho de produção científica de outros pesquisadores no campo. Posteriormente, expomos os procedimentos metodológicos utilizados na investigação empírica, cuja apresentação e análise dos dados são dispostos em seguida. Finalizamos o artigo com recomendações para futuros estudos e com as conclusões provenientes dos achados da pesquisa acerca da agência no campo científico, as quais remetem ao fenômeno que, visto cognitivamente ao nível do pesquisador, denominamos de paradoxo da capacidade da agência.

\section{Agência}

Sob o quadro estruturacionista que orienta este artigo, campo científico pode ser tratado como sistema social: relações entre atores ou coletividades, organizadas como práticas sociais regulares, continuamente reproduzidas e que manifestam propriedades estruturais cujas implicações recaem sobre condições para a continuidade desse processo recursivo (Giddens, 2003). Enquanto tal é recursivamente estruturado por meio da prática dos atores sociais.

Conforme discutem Machado-da-Silva, Guarido Filho e Rossoni (2006), tratar da estruturação do campo como reprodução de propriedades estruturais a partir das práticas dos atores significa aceitar não somente que estes sejam capazes de reproduzir, mas de criar novos padrões de interação, alterando endogenamente a sua estrutura. Isso significa que atores sociais possuem, em certa medida, a competência de controlar suas ações, de racionalizá-las, por meio de consciência 
das condições ou conseqüências implicadas, que procuram ativamente conquistar em interação com os outros (Giddens, 2001).

Desse modo, restringimo-nos a investigar os "modos como os atores sociais se apóiam em propriedades estruturais para a constituição de relações sociais [atribuindo] primazia às consciências discursiva e prática, e às estratégias de controle dentro de limites contextuais definidos" (Giddens, 2003, p. 339-340). Diante desta ênfase, investigamos o fenômeno aqui estudado sob uma ótica de análise da conduta estratégica (vide Giddens, 2003), por meio da qual os esforços analíticos remontam sobre o papel da agência humana contextualizada na estruturação do campo científico. Compreendido desta maneira, não se está admitindo a uniformidade de práticas sociais já que, em consonância com Cohen (1999, p. 416), a agência é tratada como capacidade potencial de agentes sociais produzirem variações históricas em suas próprias formas de conduta. Dessa forma, agência refere-se à capacidade de produzir um efeito: "diz respeito a eventos dos quais um indivíduo é o perpetrador, no sentido de que ele poderia, em qualquer fase de uma dada seqüência de conduta, ter atuado de modo diferente" (Giddens, 2003, p. 10-11).

No entanto Machado-da-Silva, Fonseca e Crubellate (2005) explicam que as práticas sociais dos agentes necessariamente implicam interpretação, de modo que a capacidade de monitoração reflexiva do ator social em processar cognitivamente, sob a concepção da racionalidade limitada, envolve atribuir significado a estímulos contextuais, segundo referências habituais, projeções e julgamentos. Tais aspectos convergem para a discussão apresentada por Emirbayer e Mische (1998) sobre o conceito de agência, que, por sua vez, não contrapõe a visão de Giddens (2003) acerca da agência, apenas altera o foco para o agente em particular.

Segundo esses autores, a agência humana, como processo temporalmente imerso de engajamento social, só pode ser capturada em sua complexidade quando analiticamente situada no fluxo do tempo, em que passado, presente e futuro se apresentam como dimensões distintas, porém interconectadas. Para compreender tais aspectos dessa trajetória, Emirbayer e Mische (1998) utilizam basicamente dois conceitos: um que envolve a concepção de tempo constituído como eventos emergentes, que, em poucas palavras, destaca a importância de se compreender as experiências vividas pelos agentes, já que somente a partir delas é possível destacar o significado atribuído por eles a sua própria existência; outro que delineia a consciência humana constituída a partir da sociabilidade, que nada mais é do 
que a capacidade de estar simultaneamente associado a facetas temporais e relacionais da vivência humana.

$\mathrm{Na}$ congruência desses conceitos, eventos emergentes e sociabilidade, a ação é sempre orientada pela experiência social: o passado é sempre acessado no momento da ação, com situações remotas emergindo na memória do agente diante dos diferentes contextos vivenciados por ele. No entanto as experiências passadas, que condicionam a ação presente ao mesmo tempo que servem como base de ação, também são reconstruídas pelo esforço de entender as condições causais do presente emergente, ganhando um novo significado pelo agente. Em conseqüência dessa experimentação, o esquema de interpretação do agente é modificado, de forma que há uma transformação de seus parâmetros de conduta, o que afeta suas respostas em momentos futuros.

Tal concepção remete à associação entre a dimensão temporal e aspectos relacionais, originalmente tratada por Mead (1932). Para ele, a ação é sempre condicionada por respostas a contextos entrelaçados e sobrepostos, levando os atores a construir perspectivas de forma intersubjetiva, a partir não apenas de seus pontos de vista, mas também dos de outros. Tal movimento é entendido pelo autor como capacidade deliberativa. Com efeito, somente a partir da aceitação da capacidade dos agentes de refletir sobre sua trajetória de produção social que Emirbayer e Mische (1998, p. 970) definiram agência humana como o

engajamento temporalmente construído por atores a diferentes ambientes estruturais - os contextos temporal-relacionais da ação - que, por meio da interação entre hábito, imaginação e julgamento, tanto reproduz quanto transforma aquelas estruturas, em resposta interativa aos problemas colocados por situações históricas em mudança.

Exposto dessa maneira, o conceito admite espaço para a agência criativa, aproximando-se da perspectiva estruturacionista, mesmo sem tratar explicitamente da recursividade entre ação e estrutura. Sob essa perspectiva, a noção de agência é tratada como um processo intersubjetivo, relacional e temporal (Emirbayer, 1997; Emirbayer \& Mische, 1998), que remete ao caráter transformador da ação humana. Ademais desvincula-se o conceito das concepções super ou subsocializadas de ser humano, respectivamente ligadas ao racionalismo e funcionalismo (vide Granovetter, 1985), reconhecendo sua composição tanto em termos de atividades de caráter mais rotineiro ou reprodutivo, quanto prospectivas, imaginativas e criativas (Scott, 2008).

Dessa forma, como recurso analítico, Emirbayer e Mische (1998) dividiram a agência em três dimensões complementares: interação, projetividade e avaliação 
prática. Interação diz respeito à reativação pelos atores de padrões passados de pensamento e ação, rotineiramente incorporados na atividade prática, assegurando estabilidade e ordem ao universo social, tal como os ajudando a sustentar identidades e instituições no decorrer do tempo. Já projetividade se refere à capacidade imaginativa dos atores de traçar trajetórias de ação, criativamente reconfiguradas em relação a expectativas, medos e desejos do futuro. Por fim, a dimensão prático-avaliativa aponta a capacidade dos atores de efetuar julgamentos práticos e normativos diante de possíveis alternativas de ação, em resposta a demandas emergentes, dilemas e ambigüidades de situações desenvolvidas no presente.

\section{Agência no Campo Científico: Produtividade, Centralidade e Escolha Preferencial}

Diante do exposto, o presente trabalho reconhece o pesquisador, também identificado como autor em trabalhos científicos, como agente. Dentro dos limites apresentados no presente quadro teórico de referência, isso significa considerar para fins analíticos sua capacidade de atuar com propósitos e influenciar na estrutura do conhecimento disponível no campo (Fligstein, 1999, 2001). Conforme o exposto, a capacidade de atuar em favor da construção do significado de ciência, modular práticas de pesquisa ou temáticas, bem como promover o debate intelectual, podem ser aspectos associados à agência de determinados pesquisadores no campo.

Diversos elementos contribuem para a ampliação da capacidade de agência dos pesquisadores como, por exemplo, sua atuação como orientadores de programas de pós-graduação, representantes de órgãos oficiais, membros e coordenadores de associações de pesquisadores ou de conselhos editoriais, avaliadores de artigos, entre outros. Neste trabalho, porém, o interesse maior está direcionado para indicadores que estão mais próximos da atividade diária, e não da posição que os pesquisadores ocupam no campo científico. Assim, a capacidade de um agente influenciar a construção do campo científico será analisada mediante três aspectos principais. O primeiro deles considera a relação entre produtividade, quanto ao volume de produção científica de um pesquisador, e a estrutura de cooperação em que se insere. Já o segundo aspecto dirige a atenção para a capacidade de os pesquisadores angariarem colaboradores, influenciando não apenas a estrutura de relacionamentos em que participam como também o trânsito de idéias e de informações. Por fim, o terceiro elemento considera a permanência e regularidade de um pesquisador no campo como fatores que potencializam sua capacidade de 
promover influências na produção do conhecimento. Cada um desses aspectos será brevemente tratado na seqüência.

Estudos de colaboração científica tratam do relacionamento entre pesquisadores no processo de produção do conhecimento, podendo resultar em co-autoria de trabalhos. Embora Katz e Martin (1997) expliquem que a colaboração científica não deva ser restringida à co-autoria, ela pode servir como elemento de sua representação, como destacam Glänzel e Schubert (2008).

Para fins deste trabalho, a relação entre a estrutura de cooperação no campo e produtividade possui interesse em especial. É possível admitir que pesquisadores, cuja centralidade se destaca na rede, apresentem maior capacidade de estabelecer relacionamentos de colaboração, o que por consequência implica maior potencial de influência sobre o campo, seja por meio de sua produtividade, seja a partir da influência sobre perspectivas, conceitos, idéias ou metodologias adotadas (Moody, 2004).

Diferentes estudos, conforme relatam Katz e Martin (1997) e Melin (2000), apresentam correlação entre centralidade e produtividade. Porém Lee e Bozeman (2005) advertem que o pressuposto de que a cooperação ${ }^{4}$ implica em maior produtividade ainda requer maior investigação, já que diferentes elementos podem ser associados a tal relação: divisão do trabalho, complementação de habilidades, eficiência com relação ao uso do tempo, estímulo intelectual, aprendizado, oportunidades de publicação, entre outros. Bozeman e Corley (2004) ainda sugerem que a colaboração pode ser entendida como mecanismo chave na orientação de jovens pesquisadores e estudantes de pós-graduação.

Por essas razões, a colaboração entre cientistas normalmente é referenciada como desejável (Katz \& Martin, 1997; Rodrigues \& Carrieri, 2001), assumindo até caráter normativo como demonstraram no passado Beaver e Rosen (1979). O crescimento da cooperação nas últimas décadas (Barabasi, 2005) pode ser reflexo disso: além de motivado pelo aumento dos custos, da interdisciplinaridade e da complexidade da pesquisa acadêmica, há ainda exigências de órgãos de fomento, que em alguns casos concedem recursos somente quando há interação entre pesquisadores e instituições (Lee \& Bozeman, 2005; Wuchty, Jones \& Uzzi, 2007).

Nesse processo, certos desdobramentos sugerem a ampliação do escopo da cooperação para além dos benefícios imediatos em termos de produtividade ou

\footnotetext{
${ }^{4}$ Neste trabalho, assumimos a colaboração em termos de co-autoria como unidade de análise da centralidade, o que em algumas passagens nos levou a trabalhar com os dois termos de forma intercambiável.
} 
otimização de esforço intelectual. De acordo com Katz e Martin (1997), colaboração entre pesquisadores traz benefícios potenciais quanto ao capital social e à visibilidade do conhecimento produzido, ambos os aspectos relacionados às noções de propriedade intelectual e reconhecimento acadêmico no campo científico, conforme elaborados por Merton (1996). Uma vez publicado um trabalho, aumentam as chances de influenciar o corpo de conhecimento da área em estudo, sendo tratado como referência válida para novos estudos o que, por sua vez, recompensa o pesquisador com o reconhecimento da propriedade do conhecimento por seus pares (Merton, 1996). A produtividade acadêmica, portanto, é, em si mesma, um mecanismo ligado à agência na construção do conhecimento. Entretanto sua relação com a cooperação e as implicações concernentes à difusão de idéias e acumulação de capital social potencializam a agência no sentido de garantir recursos para a organização acadêmica em torno de pesquisadores chaves no campo.

Tal aspecto remete ao segundo elemento em discussão: a preferência. Pesquisadores centralmente localizados numa comunidade podem atuar como força de difusão e legitimação do conhecimento praticado no campo (Zucker \& Darby, 1996). Eles cultivam uma gama de relacionamentos com o objetivo de prosseguir em determinadas linhas de pesquisa, ou mesmo defendê-las no debate acadêmico (Guarido Filho, Machado-da-Silva \& Gonçalves, 2009). Trata-se de propriedade ligada ao papel da agência que, nesse caso, extrapola características individuais de pesquisadores, representando estruturas sociais estratificadas de produção científica e organizadas em torno de determinados pesquisadores, cuja capacidade de atrair colaboradores em proporção significativamente maior do que outros, expressa, em termos relacionais, o que se conhece como mecanismo de escolha preferencial. Nesses casos, determinados atores detêm maiores centralidades quanto a gama de contatos com os quais se relacionam e influenciam, por meio da intermediação do fluxo de informações e idéias (Wasserman \& Faust, 1994). Relacionado a isso está o fato de que pesquisadores com destacada reputação ou que representem acesso a recursos, como, por exemplo, experiência ou conhecimento, tendem a ser mais acessados e, em função disso, aumentam seu prestígio, ao tempo que exercem influência sobre o conhecimento produzido (vide Merton, 1996 e Moody, 2004).

No entanto esses aspectos resultam, em certa medida, do histórico de atuação de um pesquisador no campo científico, cujos pesquisadores com atividade continuada tendem a se destacar - e aqui chegamos ao terceiro componente analítico que sustenta o presente estudo. Pesquisadores continuantes são aqueles que possuem 
um retrospecto de permanência e regularidade em termos de produção no campo (Braun, Glänzel \& Schubert, 2001), os quais exercem a intermediação de relacionamentos de cooperação entre pesquisadores mais jovens e outros já dispostos no campo, atuando também como disseminadores do conhecimento e orientadores da prática científica (vide Wagner \& Leydesdorff, 2005). Sendo assim, pesquisadores continuantes tendem a ter maior capacidade de agência que os demais.

Assim, tomando-se os fundamentos teóricos a respeito da agência e suas implicações na construção do conhecimento científico com base nos elementos aqui discutidos, realizamos o estudo sobre o campo da pesquisa em organizações e estratégia no Brasil, cujos procedimentos metodológicos são explicados a seguir.

\section{Procedimentos Metodológicos}

Dados e amostra. Utilizamos a pesquisa documental como estratégia de coleta de dados da produção científica das áreas de estudos organizacionais e estratégia em organizações no Brasil. Adotamos como unidade amostral os artigos científicos publicados nas áreas de organizações e estratégia em anais dos congressos da ANPAD - Associação Nacional de Pós-Graduação e Pesquisa Administração (EnANPAD, EnEO e 3Es) e periódicos científicos classificados como A Nacional em 2006 pela agência brasileira de acreditação de programas de pós-graduação stricto sensu [CAPES]: Revista de Administração Contemporânea (RAC), Revista de Administração de Empresas (RAE), Revista de Administração de Empresas Eletrônica (RAE-E), Revista de Administração (RAUSP), Organizações \& Sociedade (O\&S) e Revista de Administração Pública (RAP). Todos os periódicos e congressos mencionados adotam o procedimento de double blind review de avaliação por pares.

Identificamos 2.332 trabalhos publicados no período compreendido entre $1997 \mathrm{e}$ 2005, o que proporcionou uma população de 2.072 pesquisadores, integralmente avaliados. O método de pesquisa empregado foi quantitativo apoiado sobre a análise de redes sociais e procedimentos estatísticos. Os dados foram tabulados considerando os relacionamentos de co-autoria entre pesquisadores, registrados em matrizes quadradas que possibilitaram a construção de redes de cooperação na produção científica. A análise dos dados foi realizada por meio dos softwares UCINET 6, PAJEK e Statistica.

Por considerar que delineamentos longitudinais são necessários para exprimir a relação entre agência e estrutura, em conformidade com Emirbayer e Mische 
(1998), Giddens (2003) e Machado-da-Silva, Fonseca e Crubellate (2005), avaliamos a trajetória dos pesquisadores e do campo em períodos de três anos: (i) de 1997 a 1999, (ii) de 2000 a 2002 e (iii) de 2003 a 2005. A escolha pela divisão trienal justifica-se por evitar efeitos da sazonalidade provocada pela realização de alguns eventos que ocorrem bienalmente, além de propiciar comparação real com base em períodos de mesma amplitude, favorecendo a interpretação dos resultados. Com as redes configuradas em períodos, foi possível analisar as características descritivas do campo e dos pesquisadores.

Operacionalização da agência. Para a compreensão do papel da agência na construção do conhecimento científico, foram analisadas as relações de cooperação, mais propriamente de co-autoria na produção de trabalhos, e o volume de publicações dos pesquisadores destas áreas tendo em vista os elementos tratados no referencial teórico: centralidade, produtividade e escolha preferencial.

Primeiramente, realizamos a análise da cooperação. A partir dos relacionamentos de cooperação entre pesquisadores, calculamos medidas de centralidade a fim de avaliar se seu posicionamento na rede exerce influência na produtividade, mensurada de acordo com o volume de artigos publicados. Complementarmente a Lee e Bozeman (2005), que consideraram somente a cooperação direta, por meio da centralidade de grau (degree centrality), adicionamos, para esse fim, as medidas de centralidade de intermediação (betweenness centrality) e de poder (power centrality), sendo esta última desenvolvida originalmente por Bonacich (1987). A primeira delas avalia a capacidade de angariar colaboradores. Já a segunda, possibilita avaliar se a capacidade de pesquisadores atuarem como intermediadores de outros não diretamente relacionados influencia a produtividade. Por fim, a medida de poder de Bonacich (1987), correntemente interpretada como uma medida estrutural de prestígio, foi utilizada para verificar se pesquisadores que se relacionam, direta ou indiretamente, com outros que apresentam grande número de colaboradores, apresentam maior produtividade.

Métodos de Análise. Considerando essas três medidas de centralidade, utilizamos o modelo de regressão Ridge, indicado quando há multicolinearidade entre as variáveis $^{5}$, pois ajusta o tamanho do efeito de cada uma delas (Hair, Anderson, Tatham \& Black, 2005). Foram aceitos como significativos, os resultados que se enquadraram no intervalo de confiança de $95 \%(p<0,05)$. Além disso, ilustramos

\footnotetext{
${ }^{5}$ Ressalta-se que o uso do modelo Ridge é relevante, pois possibilita controlar a multicolinearidade dos dados, possibilitado melhor ajuste em função de serem correlacionados e interdependentes. Para efeitos de validação, avaliamos também a relação entre as variáveis por meio do método Bootstrap, presente no UCINET, que é adequado para dados relacionais (vide Hanneman e Riddle, 2005), corroborando a análise do modelo Ridge.
} 
graficamente a relação entre produção científica e centralidade de grau por meio de gráfico de rede desenvolvido com auxílio do software PAJEK.

Em relação à escolha preferencial, seguimos as orientações de Barabasi e Albert (1999). Os pesquisadores foram agrupados em tabelas de freqüência em relação ao grau de cooperação, posteriormente dispostas em diagramas de dispersão. A partir desse ponto, três procedimentos foram realizados. Inicialmente, verificamos o ajuste da distribuição de freqüência dos laços à função potência, comparando seu coeficiente de determinação com o calculado para outros tipos de funções: exponencial, quadrática e logarítmica. Observamos que, a fim de assegurar maior rigor nesse processo, optamos por analisar diretamente o ajuste dos dados à função potência, o que difere do realizado por estudos anteriores, que previamente fizeram a transformação logarítmica das medidas de freqüência e grau para a posterior verificação de ajuste linear (vide Moody, 2004; Newman, 2001; Wagner \& Leydesdorff, 2005).

No segundo procedimento, seguindo Wagner e Leydesdorff (2005), exportamos os dados de freqüência e de grau para o software LOTKA (Rousseau \& Rousseau, 2000) para, novamente, avaliar o ajuste da distribuição por um método mais robusto, o teste de Kolmogorov-Smirnov, que possibilita a avaliação da significância da escolha preferencial. Por último, avaliamos o valor do expoente da função potência, visando compreender a tendência de afiliação; admitimos que expoentes entre -1 e -2 indicam a existência de concentração de nós com grande número de laços, conforme White, Owen-Smith, Moody, e Powell (2004), que associaram esses expoentes a redes em que existem vários integrantes com grande número de laços. Já expoentes com valores entre 2 e 3 (identificados originalmente por Barabasi \& Albert, 1999) tendem a surgir em redes mais centralizadas.

Objetivando diferenciar os pesquisadores em relação à sua presença no campo, utilizamos a forma adaptada das categorias apresentadas por Braun, Glänzel, e Schubert (2001), conforme realizada por Guarido Filho, Machado-da-Silva e Gonçalves (2009). Nessa classificação, os pesquisadores foram categorizados como entrantes, one-timers, transientes, continuantes ou retirantes em função do volume e regularidade de sua publicação na área ao longo de todo o período investigado. Para tanto, consideramos o número de artigos publicados por um pesquisador, o momento em que sua produção ocorreu e a distribuição da produção ao longo dos anos. Seguindo Guarido Filho, Machado-da-Silva e Gonçalves (2009), foram considerados continuantes os pesquisadores que publicaram em 5 ou mais diferentes anos do período em estudo, com intervalo de não mais do que dois anos 
entre cada publicação e pelo menos uma nos últimos três anos. Transientes possuem mais de uma publicação distribuídas ao longo do período analisado em não mais do que quatro anos diferentes, sendo ao menos uma nos últimos três anos. Entrantes, por sua vez, apresentam mais de uma publicação, exclusivamente, em um ou mais anos diferentes dos últimos três anos do período em estudo, enquanto one-timers possuem apenas uma única publicação em todo o período analisado. Por fim, retirantes são os pesquisadores com mais de uma publicação em um ou mais diferentes anos, mas sem publicações nos últimos três anos.

Esse procedimento resultou em classificação estática, considerada aqui adequada por estarmos interessado na atividade agregada dos pesquisadores. Além disso, evita as limitações da classificação dinâmica, que fica restrita apenas aos períodos centrais da janela temporal estudada. Após a classificação, as diferentes categorias foram comparadas com relação às médias de centralidade de grau, intermediação e poder e, ainda, quanto ao número de artigos publicados. Utilizamos o teste ANOVA para esse fim, de modo a avaliar diferenças significativas entre as categorias.

Por fim, para entender a trajetória de construção do conhecimento dos pesquisadores mais proeminentes no campo, realizamos análise de conteúdo dos artigos publicados por eles. Para tanto, definimos uma amostra envolvendo somente os pesquisadores continuantes, que foram selecionados a partir do procedimento de Braun, Glänzel, e Schubert (2001), que foi adaptado por Guarido Filho, Machado-da-Silva e Gonçalves (2009), nos termos já explicados anteriormente. Adicionalmente, também definimos outro conjunto de casos tendo como unidade de análise os grupos atuantes no campo, que foram selecionados a partir da medida de coesão $n$-clan. Em poucas palavras, a medida $n$-clan é muito semelhante a n-clique, que é um grupo cuja distância geodésica entre dois nós pertencentes a ele é menor ou igual a $n$, no qual $n$ é o caminho máximo em que membros de um clique podem estar conectados (Scott, J. 2000). A diferença reside no diâmetro da $\mathrm{n}$-clan, que deve ser menor ou igual a $\mathrm{n}$ (Wasserman \& Faust, 1994). Entre as várias possibilidades de diâmetro, selecionamos um $n$ igual a 2 , um 2-clan, pois como apontam Hanneman e Riddle (2005), tal medida é mais adequada para identificar grupos sociais. Na sequência, identificamos todos os artigos publicados por cada um dos pesquisadores, assim como pelos grupos, separando elas em três períodos de tempo: 1997-1999; 2000-2002; 2003-2005. Depois disso, seguindo os procedimentos desenvolvidos por Bardin (1977), adotamos o critério de categorização semântico por "milha", pelo qual a categorização temática é feita a posteriori, como resultante da análise do conteúdo científico dos artigos, mais especificamente o quadro teórico de referência, o 
fenômeno pesquisado e, em alguns casos, a metodologia. Em face da classificação dos artigos perante os pesquisadores e grupos que produziram, conseguimos avaliar quais deles persistiram em suas trajetórias ou não, comparando diferentes períodos de produção.

\section{Resultados: Agência no Campo da Pesquisa}

O campo da pesquisa em organizações e estratégia cresceu significativamente no Brasil, entre os anos de 1997 e 2005. O volume de artigos publicados nas fontes investigadas passou de 338 entre 1997 e 1999 para 736 no triênio seguinte, até alcançar 1.258 trabalhos produzidos ao longo dos anos de 2003 a 2005, totalizando 2.332 artigos em todo o período. Notamos esse crescimento também quanto ao número de pesquisadores participando no campo. Dos 2.072 pesquisadores identificados no período investigado, apenas 387 estiveram presentes no primeiro triênio (1997-1999), montante bastante inferior aos 1.258 encontrados no último (2003-2005).

Em termos estruturais, conforme representado na Figura 1, observamos uma configuração com baixa densidade, menor do que $1 \%$ do total de laços possíveis, e alta fragmentação. Apesar disso, a cooperação entre pesquisadores, em termos de co-autoria, cresceu: de 1,55 laços no primeiro período, para 2,07 laços no último, em convergência com o já constatado por Barabasi (2005) e Lee e Bozeman (2005) que apontaram esse fenômeno como cada vez mais frequente. Contudo verificamos que o coeficiente de agrupamento se manteve estável ao longo do tempo, bem como a distância média entre os pesquisadores, mesmo diante do crescimento acentuado da área.

Na Figura 1, o tamanho dos nós representa o volume de colaboradores diretos que um pesquisador apresentou ao longo do período, isto é, sua centralidade de grau. As diferentes cores, por sua vez, indicam componentes da rede, ou seja, conjuntos de pesquisadores com ligações entre si, mas não conectados aos demais. Como se pode notar, apesar da fragmentação verificada em todos os momentos, na maioria dos componentes há pesquisadores com maior grau de relacionamento (nós maiores) e que funcionam como elos entre pesquisadores, influenciando a coesão da rede. Isso é mais evidente nos componentes de maior tamanho. Admitindo-se que o posicionamento de tais indivíduos favoreça a divulgação de idéias, assim como exerça influência intelectual em outros pesquisadores, em concordância com Friedkin (1998), é plausível supor que eles possuam maior capacidade de agência. Em face disso, procuramos verificar empiricamente se a centralidade dos pesquisadores na rede de colaboração influencia sua produtividade. 


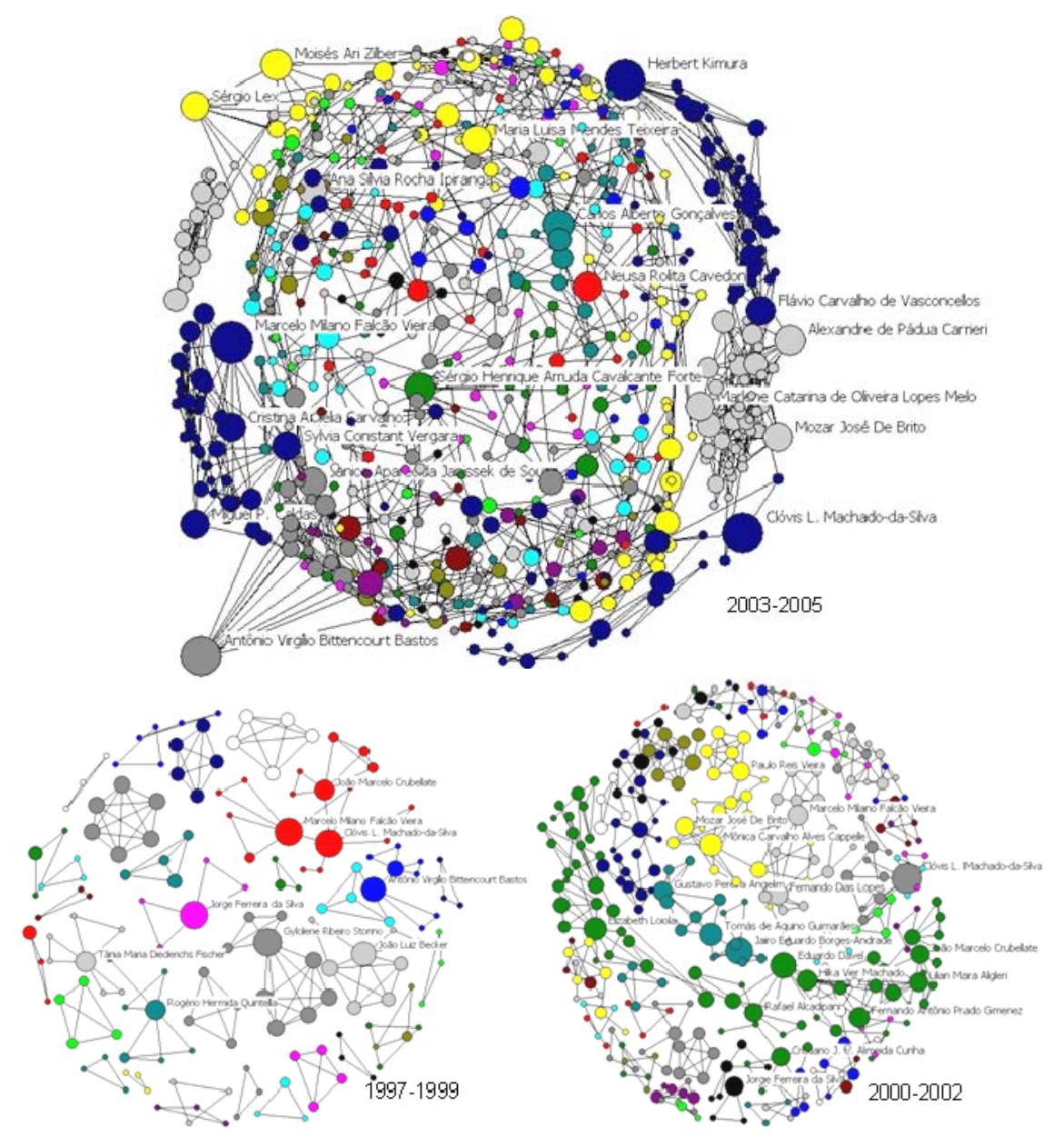

Figura 1. Relações de Co-autoria por Período.

$\mathrm{Na}$ Tabela 1, apresentamos os resultados da análise de regressão, para a qual os tipos de centralidade foram considerados variáveis independentes e a produtividade, variável dependente. De imediato, vale ressaltar que, em razão deste estudo tomar como arcabouço teórico os elementos da teoria da estruturação, entendemos que a reprodução de sistemas sociais, como é o caso do campo científico, deve ser tratada como desempenho qualificado da agência humana, e não simplesmente como uma série mecânica de processos (Giddens, 1978). Por conseguinte, rejeitamos a possibilidade de interação funcional da estrutura de relações e a produção científica dos pesquisadores, o que implica não se atribuir significado causal às relações desses dois elementos. Por essa razão, apesar do uso de técnica de dependência, a regressão linear, nossa avaliação trata do imbricamento entre as variáveis, numa relação de dualidade entre produção científica e estrutura de relações.

Os coeficientes padronizados dispostos na Tabela 1 indicaram forte relação entre as variáveis, de pouco mais de 59\%, quando considerados de forma agregada todos 
os períodos em análise. Na comparação entre os triênios, o que observamos foram coeficientes de explicação mais elevados nos dois primeiros, ficando em torno de $40 \%$, com redução para $24,4 \%$ no último período, efeito que pode estar associado à entrada de muitos pesquisadores com laços de cooperação, mas somente em poucos artigos.

\begin{tabular}{lcccc}
\hline Modelo: Ridge Regression & \multicolumn{4}{c}{ Período } \\
\cline { 2 - 5 }$(\lambda=0,10)$ & $1997-1999$ & $2000-2002$ & $2003-2005$ & $1997-2005$ \\
\hline Degree (grau) & $0,422 * * *$ & $0,500 * * *$ & $0,182 * * *$ & $0,370 * * *$ \\
Power (poder) & $-0,232 * * *$ & $-0,140 * *$ & $0,405 * * *$ & $0,131^{* * *}$ \\
Betweenness (intermediação) & $0,423 * * *$ & $0,338 * * *$ & $-0,00018$ & $0,347 * * *$ \\
\hline $\mathrm{R}^{2}$ & $38,87 \%$ & $39,52 \%$ & $24,40 \%$ & $59,72 \%$ \\
Significância & $<0,001$ & $<0,001$ & $<0,001$ & $<0,001$ \\
\hline Número de Casos & 387 & 785 & 1445 & 2072 \\
\hline
\end{tabular}

$* * * \mathrm{p}<0,001 \quad * * \mathrm{p}<0,01$

Tabela 1. Relação entre Centralidade e Produtividade dos Pesquisadores.

Vale destacar que, entre as variáveis independentes, a centralidade de grau, que indica o número de colaboradores vinculados a um pesquisador, foi a que mais exerceu influência na produtividade. A Figura 2 ilustra essa questão: quanto maior a centralidade de grau de um pesquisador, mais no alto da rede ele se posiciona; quanto mais artigos publicados pelo pesquisador no período, maior o tamanho do nó. Em outros termos, os resultados apontam que o número de co-autores mantidos por um pesquisador está positivamente relacionado com sua produtividade.

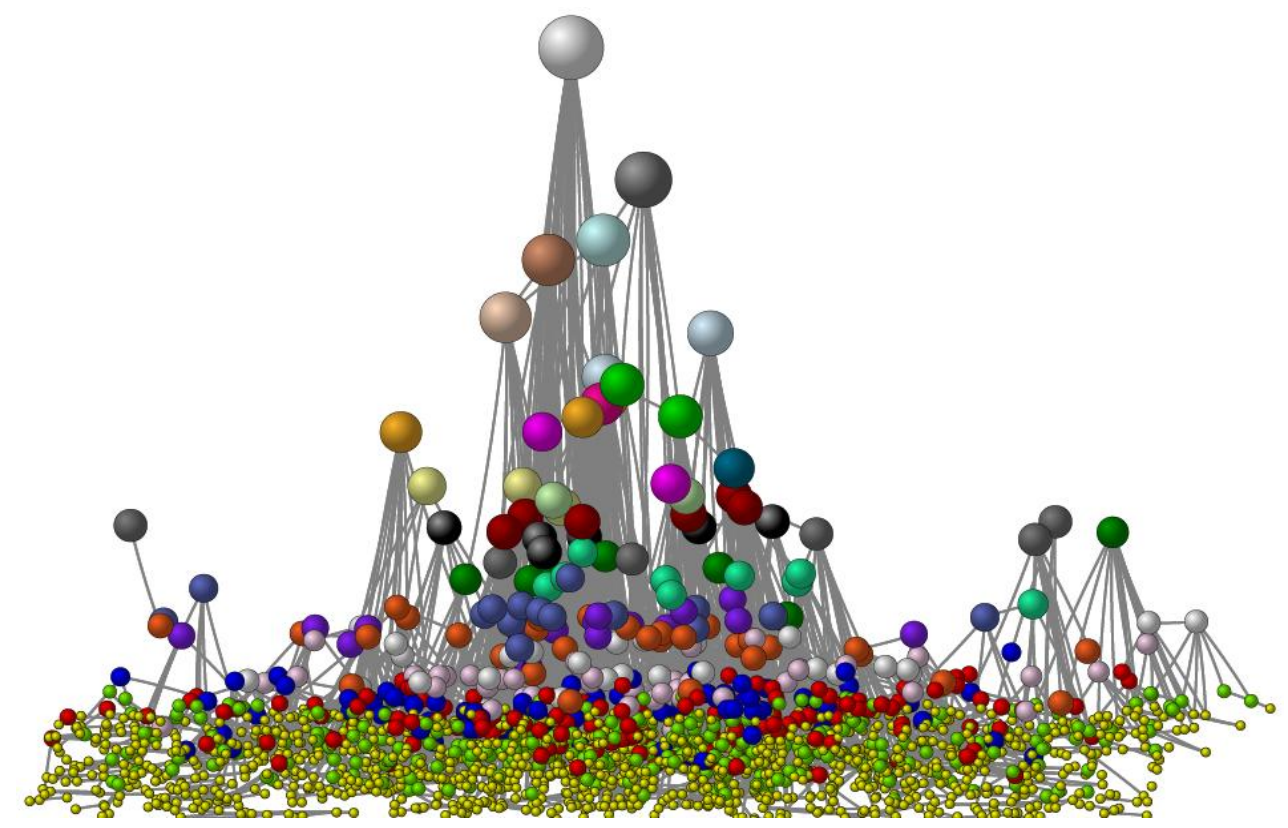

Figura 2. Relação entre Centralidade de Grau e Produção dos Pesquisadores. 
Apesar disso, conforme indicado na Tabela 1, a medida de centralidade que mais aumentou sua influência foi a de poder (power centrality), que envolve o noção de prestígio dos pesquisadores. Isso pode ser explicado de duas formas. Primeiramente, por esse indicador considerar o número de laços diretos e indiretos daqueles autores com os quais o pesquisador se relaciona, ele aponta que a produtividade está associada, em termos estruturais, ao prestígio dos co-autores, fazendo referência a capacidades cumulativas do agente, que usufrui dos vínculos mantidos por outros pesquisadores com os quais produz e, assim, aumentando seu potencial de produção científica. Em níveis mais amplos de imersão do que os de colaboração direta, tal efeito se refere à formação de capital social como demonstraram Uzzi \& Lancaster (2004).

Em segundo lugar, esse efeito também pode ser compreendido a partir de sua associação com o desenvolvimento do campo da pesquisa em termos de estrutura de relações. Num primeiro momento, pesquisadores novatos tendem a entrar no campo quase exclusivamente por meio de relações com pesquisadores mais experientes, em sua maioria professores (vide Rossoni \& Machado-da-Silva, 2008; Guimera, Uzzi, Spiro \& Amaral, 2005) que, por essa razão, têm incrementada sua capacidade de intermediação. No entanto, com o crescimento do campo e com o aumento da cooperação, muitos daqueles novatos permanecem na rede, desenvolvendo novas relações de co-autoria com outros pesquisadores. Por causa disso, a contribuição que inicialmente provocaram na capacidade de intermediação de seus pares mais experientes se reduz. Em contrapartida, proporcionam aumento da centralidade de poder daqueles que uma vez possibilitaram seu acesso ao campo, devido à formação de linhas de prestígio em níveis mais amplos.

Conforme é possível observar na Tabela 1, esse mecanismo pode ser verificado empiricamente: na medida em que decresce o coeficiente de explicação da centralidade de intermediação, de 0,443 no primeiro período para próximo de zero no último, aumenta a força da centralidade de poder como variável explicativa para a produtividade.

Em face de tais resultados, a capacidade de agência pode ser interpretada como fruto dessa transição de influência, de intermediação para poder, na medida que o campo da pesquisa em organizações e estratégia vai se estruturando. A ideia subjacente é a de que os canais de influência se ampliam, propiciando não apenas a influência nos relacionamentos diretos, mas também na construção do conhecimento. Todavia, vale destacar que não se deve reduzir esse movimento a manobras estratégicas promovidas por certos pesquisadores, de tal modo que, 
apesar do componente intencional estar presente, trata-se de consequências impremeditadas da ação ao longo da estruturação do campo (vide Giddens, 2003). Esse fenômeno é próximo ao que Wagner e Leydesdorff (2005) intitulam sistemas auto-organizados, nos quais mecanismos de afiliação entre pesquisadores, em nível micro, repercutem na estruturação da rede global (nível macro).

Dando continuidade à investigação do papel da agência, avaliamos a existência de mecanismo do tipo escolha preferencial no campo (Barabasi \& Albert, 1999). A ideia de escolha preferencial está diretamente ligada à de estratificação na ciência, considerando a dimensão relacional entre pesquisadores na produção científica (Rossoni \& Guarido Filho, 2009). Afirmar que o mecanismo de escolha preferencial está presente em redes de colaboração destaca a capacidade de alguns poucos pesquisadores atrair número de colaboradores significativamente maior que a maioria dos demais, o que implica em participação destacada de certos pesquisadores no campo. Estruturalmente, implica numa distribuição do tipo potencial inversa (power-law) calculada a partir da centralidade de grau dos pesquisadores, representando sua capacidade de intermediar o fluxo de informações entre pesquisadores não diretamente conectados.

Esse mecanismo pode ser visualizado a partir da Figura 3, que, no eixo horizontal, traz o volume de laços (centralidade de grau) e, no eixo vertical, o número de pesquisadores. Observa-se que o número de pesquisadores reduz, gradativamente, à medida que cresce o número de laços. Tal situação reforça o que já mencionamos anteriormente: a condução de pesquisadores entrantes por outros mais centrais e já atuantes no campo, situação que, para esses últimos, provoca cada vez maior concentração de laços (Newman, 2001).

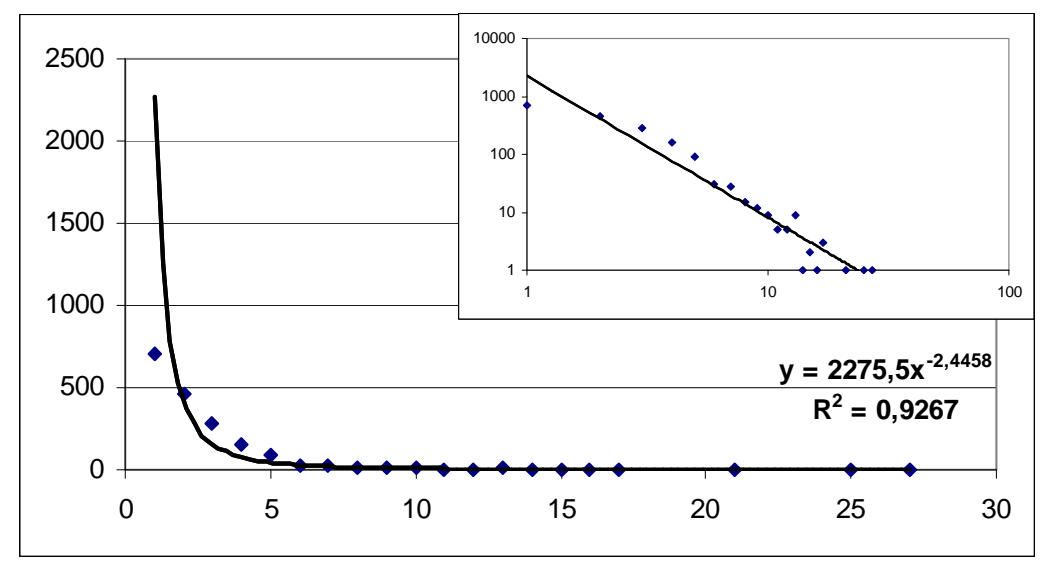

Figura 3. Relação de Escolha Preferencial.

A existência de escolha preferencial, conforme exposto nos procedimentos metodológicos, foi validada pelo fato de a função potência ter demonstrado melhor 
ajuste aos dados do que outras distribuições ( $R^{2}$ de $92,7 \%$ ), embora o teste de Kolmogorov-Smirnov não tenha sido significativo. No presente caso, essa distorção pode ser devido a certas características das práticas de colaboração da comunidade acadêmica brasileira, em que constatamos uma frequência elevada de pesquisadores com uma única publicação produzida em co-autoria. Isso implica em distorção no ajuste da função potência.

Adicionalmente, considerando-se o expoente da função, de valor -2,45, podemos inferir a existência de outros mecanismos atuantes no estabelecimento de relações entre pesquisadores no campo da pesquisa em organizações e estratégia, dos quais dois merecem destaque. O primeiro deles converge com o resultado encontrado por Moody (2004): assim como no campo internacional da sociologia, a pesquisa em organizações e estratégia no Brasil tem apresentado especialização dos pesquisadores em conteúdos específicos, o que nos habilita a afirmar que estamos diante de um mecanismo de estruturação do campo. Já o segundo, diz respeito a evidência de um número pequeno de pesquisadores com elevada quantidade de laços de colaboração, acarretando na maior centralização do campo.

Em face do exposto, entendemos que estruturas estratificadas com base no mecanismo de escolha preferencial, por contar com a presença de pesquisadores em posição destacada no fluxo relacional, evidenciam aspectos ligados à agência no campo, especialmente em razão de sua influência no sistema de comunicação acadêmica, modelando o conhecimento e também formando multiplicadores.

No entanto vale considerar que essas questões podem sofrer influência do tempo de atividade de um pesquisador no campo, bem como da regularidade de produção. Por essa razão, os pesquisadores foram classificados em cinco categorias com objetivo de compará-las com relação às medidas de centralidade e produtividade. $\mathrm{Na}$ Tabela 2, apresentamos os valores médios de cada categoria com relação às centralidades de grau, poder, intermediação e produção.

\begin{tabular}{lrcccc}
\hline & \multicolumn{1}{c}{ Casos } & Grau & Power & Betweenness & Produção \\
\hline Continuantes & $97(5 \%)$ & 7,89 & 19,41 & $13.957,12$ & 11,91 \\
Transientes & $288(14 \%)$ & 3,25 & 7,84 & $2.508,92$ & 3,73 \\
Retirantes & $127(6 \%)$ & 2,15 & 6,34 & $1.843,62$ & 2,51 \\
Entrantes & $243(12 \%)$ & 2,75 & 6,86 & 757,26 & 2,62 \\
One-Timers & $1.317(64 \%)$ & 1,54 & 4,24 & 0,00 & 1,00 \\
\hline Total & 2.072 & 2,25 & 5,89 & $1.203,94$ & 2,17 \\
\hline Valor do F & & 302,2 & 248,0 & 118,6 & $1.011,4$ \\
Significância & & $<0,001$ & $<0,001$ & $<0,001$ & $<0,001$ \\
\hline
\end{tabular}

Tabela 2. Média dos Indicadores por Categoria. 
Como se pode notar, dos 2.072 pesquisadores há 97 continuantes, categoria que, diante dos resultados, se mostrou especialmente relevante para este estudo. Este grupo de pesquisadores apresentou média significativamente maior que as demais categorias quanto à colaboração (7,89 laços), intermediação, produção (11,91 artigos) e melhor posicionamento na estrutura da rede (power). Tendo em vista, a relação entre medidas de centralidade e produção, constatadas anteriormente, é possível considerar que pesquisadores continuantes, em sua maioria, são responsáveis por desencadear escolhas preferenciais no campo, resultado convergente com teorias sobre vantagem cumulativas (Lee \& Bozeman, 2005).

No caso aqui estudado, continuantes possuem papel especial, atuando como canalizadores/distribuidores de relações entre pesquisadores de categorias distintas, em consonância com Guarido Filho, Machado-da-Silva e Gonçalves (2009). Nessa linha de argumentação, aceitar a existência de pesquisadores mais centrais (e continuantes) implica considerá-los força de difusão e de legitimação do conhecimento praticado no campo, diante do capital social que construíram (Zucker \& Darby, 1996). Como tais, podem apresentar a preocupação em cultivar relacionamentos que possam dar prosseguimento aos desenvolvimentos intelectuais nos quais se encontram envolvidos, ou de forma menos intencional, pesquisadores mais centrais estão envolvidos num grande número de relacionamentos, ativando uma gama de contatos, de modo a ganharem proeminência ou visibilidade enquanto canal influente de informação (Wasserman \& Faust, 1994). Diante disso, pesquisadores com essas características são capazes de exercer certa liderança, no sentido de promover a difusão de idéias, mobilizar estruturas e gerar produção científica (Guarido Filho, Machado-da-Silva \& Gonçalves, 2009), em suma: maior capacidade de agência.

Esses resultados também convergem com Guarido Filho (2008), que constatou que a estratificação revelada por meio de escolha preferencial tem forte associação com o papel de continuantes na formação do campo da teoria institucional no Brasil. Esses pesquisadores atuaram mais consistentemente na estruturação de grupos de pesquisa e, por conseqüência, na estrutura intelectual do campo, formando redes de seguidores e atuando como proliferadores de conhecimento e intermediadores de novas relações. Reforçando os argumentos de que a colaboração influencia a construção do conhecimento científico, Acedo, Barroso, Casanueva e Galán (2006) e Wuchty, Jones e Uzzi (2007) observaram que artigos co-autorados tendem a ser mais citados, o que implica em possibilidade de que pesquisadores mais bem posicionados apresentem maior influência no campo no que se refere ao 
direcionamento daquilo que é utilizado como aporte teórico trabalhos de outros pesquisadores.

A constatação de que continuantes possuem participação diferenciada na produção do conhecimento, mediante laços de orientação e difusão de perspectivas ou linhas de pesquisa, também revela indícios de que a imersão em grupos sociais condiciona o que é legitimamente aceito no campo da pesquisa. Esses achados coadunam-se com as idéias desenvolvidas por Machado-da-Silva e Rossoni (2007) e Guarido Filho (2008). Esses autores observaram que relacionamentos, em termos de agrupamentos, servem como mecanismos de homogeneização de temas e perspectivas de grupos no campo da pesquisa em estratégia. Os autores constataram que tais grupos servem como meio de geração de identidade e, com base nisso, verificaram que conjuntos de pesquisadores tendem a apresentar certas trajetórias de pesquisa, cujas características se aproximam da noção de paradigmas, conforme tratada por Kuhn (1978).

Até este momento, nossas análises dedicaram-se ao entendimento da influência da agência sobre a estruturação do campo. Todavia, há considerações adicionais que merecem ser discutidas quando direcionamos atenção ao nível do agente e que vão para além daquelas ligadas às consequências impremeditadas da ação e da estrutura social (Giddens, 2003). É nesse ponto que lançamos nosso último argumento e que orientou a parte final da análise dos dados: de que não somente a estrutura condiciona a agência, mas a própria agência tem caráter condicionante sobre a prática de pesquisa quando analisada no nível do pesquisador.

A partir de procedimentos de análise de redes (medida n-clan), identificamos diversos grupos no campo em estudo durante os períodos investigados: 9 no primeiro, 28 no segundo e 66 no terceiro. Daqueles grupos que persistiram ao longo do tempo, observamos que preservavam perspectivas teóricas e temáticas, trazendo indícios de que tais grupos influenciaram as práticas de pesquisa. Vale destacar que esses grupos também apresentaram pesquisadores continuantes, que atuaram como guia para pesquisadores entrantes.

Esse fato também foi evidenciado por Guarido Filho, Machado-da-Silva e Gonçalves (2009) e Rossoni e Machado-da-Silva (2008), que verificaram que professores e pesquisadores com maior produção tendem a conduzir as relações de outras categorias no campo. No caso da presente pesquisa, esses indivíduos identificados como continuantes, pelas razões expostas anteriormente, possuem capacidade de influenciar seus grupos, e até o campo como um todo, provavelmente em proporção maior do que são influenciados. Dessa forma, podem ser vistos como 
possuidores de maior capacidade de agência. No entanto a capacidade de influenciar o campo também condiciona suas ações, como veremos a seguir.

Avaliando qualitativamente a trajetória dos pesquisadores continuantes no campo da pesquisa em organizações e estratégia, observamos que a grande maioria deles manteve as mesmas perspectivas em termos teóricos e até metodológicos. Por exemplo, pesquisadores que atuam no campo da pesquisa em estratégia numa perspectiva econômica e utilitarista, tendem a suplantar outras perspectivas na análise de diferentes problemas. Por sua vez, pesquisadores com formação pautada em correntes interpretativistas, tendem a avaliar fenômenos econômicos numa lógica menos orientada ao aspecto utilitarista-racional. Já pesquisadores da corrente institucionalista adotam um quadro de referência próprio que, ainda quando combinado com outros referenciais, é utilizado no estudo de uma variedade de fenômenos organizacionais. De forma mais ampla, mesmo durante períodos em que havia predominância de certas temáticas de estudo, isso não acarretou em mudança de perspectiva entre os continuantes, que preservaram seu quadro de referência, salvo raras exceções.

Tais situações indicam que indivíduos que atuaram como condutores de suas abordagens de interesse tendem a não levar em consideração outras perspectivas. Assim, limitavam suas escolhas futuras a partir de quadros analíticos construídos no passado. Recapitulando Emirbayer e Mische (1998), agência é sempre um engajamento espaciotemporal; por isso, acreditamos que os elementos projetivos da agência, a partir do momento que pesquisadores apresentam maior sucesso, em termos de possibilidade de expor seu trabalho no campo, tendem a ser cada vez mais rotinizados, condicionando suas atividades prático-avaliativas.

\section{Conclusões}

O presente estudo procurou resgatar o papel da agência na construção do conhecimento científico. Para tanto, avaliamos sua influência considerando três aspectos: relação entre centralidade e produtividade, avaliação da escolha preferencial e análise de pesquisadores continuantes.

Nossos resultados evidenciam que a produtividade dos pesquisadores está associada à sua capacidade de articular laços de colaboração, de promover a intermediação e de se posicionar na rede de co-autoria de modo a manter vínculos com outros pesquisadores de maior prestígio. Ademais, constatamos que a pesquisa em organizações e estratégia no Brasil se organiza em torno de um número reduzido de pesquisadores, geralmente classificados como continuantes, 
que concentram um número de colaboradores em escala bastante superior aos demais, o que caracteriza a ocorrência de escolha preferencial.

Esses continuantes ainda se destacaram por atuarem como condutores do conhecimento produzido no campo, seja na recepção de novatos ou na organização de agrupamentos de pesquisadores. Além disso, exercem influência sobre a difusão de linhas de pensamento, temáticas e metodologias. Em conjunto, nossos achados apontam a capacidade de agência de alguns pesquisadores na estruturação do campo, o que, entretanto, não significa afirmar que ele seja produto exclusivo de suas ações intencionais.

Inferindo um significado mais amplo ao fenômeno da agência no campo da pesquisa aqui estudado, podemos entender que indivíduos mais bem posicionados em uma estrutura relacional têm maior capacidade de causar efeito sobre o campo do que indivíduos não tão bem posicionados. Tendo em vista os resultados desta pesquisa, concluímos que a existência de uma estrutura social de forma alguma pode ser vista somente como limitadora da ação; pelo contrário, sua existência também habilita a ação dos agentes, como teoricamente já apontou Giddens (2003). Sendo assim, qualquer concepção teórica que veja a estrutura estritamente como restrição não poderá compreender adequadamente a relação entre estrutura e agência. Ao considerar apenas a dimensão da restrição, limita-se a visualizar tão somente parte insuficiente de fenômeno tão complexo.

Para tornar esse aspecto mais claro, destacamos dois pontos. Primeiramente, buscamos frisar que agência não pode ser equiparada a liberdade pura de ação nem a ausência de uma estrutura social. Portanto é um erro pensar que quanto maior o condicionamento da estrutura social, menor a capacidade de um agente produzir efeito. Pelo contrário, no caso utópico de não se considerar a existência de estrutura social, a ação sempre será puramente individual, já que não existirá ação social entre indivíduos, e, em consequência, sociedade. Nessa linha de raciocínio, a existência de agência pressupõe a existência de estrutura.

Em segundo lugar, se a estrutura simultaneamente apresenta essas duas propriedades sobre a ação, restringindo e habilitando a capacidade de agir, é problemático afirmar a respeito de algum sentido paradoxal inerente à imersão social da agência, como assim o fazem, por exemplo, Battilana (2006) e Seo e Creed (2002). Ao que nos consta, pelo menos num sentido de contradição, não há nada na dualidade da estrutura que bloqueie a capacidade transformadora do agente. 
Assim, em complementação à literatura sobre agência e estrutura, os resultados do presente estudo demonstram que entre os condicionantes da ação vale chamar a atenção para o que denominamos de paradoxo da capacidade de agência, que se situa no plano estritamente cognitivo. A essência desse paradoxo reside no fato de que quanto mais capaz um agente, mais restritas são suas projeções futuras, o que afeta a amplitude de sua capacidade deliberativa. Nessa linha de raciocínio, é provável que, quanto maior o nível de imersão de um agente em um campo, maior a perspectiva temporal de projeção no qual ele se engaja, já que passa a compreender mais profundamente tanto a sua trajetória no campo, quanto a de outros agentes.

Deste modo, ao tempo que sua presença, atuação e posicionamento o habilita a influenciar a ação de seus pares e a estrutura intelectual da área, também faz com que elabore quadros de referência considerados seguros para a sua própria ação de produção de conhecimento científico. Paradoxalmente, esse aspecto também define uma espécie de fronteira cognitiva, pois o conhecimento desses mecanismos o leva ao estabelecimento de relações causais mais sedimentadas, no sentido de vincular ações à trajetória social e intelectual vivida no campo, que, sob o ponto de vista do agente, pode acarretar maior conformidade. Portanto o fato de o agente apresentar maior capacidade projetiva também o leva a agir e a pensar de forma mais habitual.

Em face do exposto, se o agente busca soluções para problemas de forma mais rotineira porque confia em sua capacidade deliberativa, a probabilidade dele não mais racionalizar aspectos anteriormente ausentes na sua rotina pode levá-lo a deixar de perceber elementos que se tornaram relevantes, afetando sua capacidade projetiva.

Contudo, mesmo tendo dado ênfase à faceta cognitiva da agência, neste trabalho não atribuímos prioridade à agência individual, mas à reciprocidade entre os atores sociais que em interação constroem e reconstroem os sistemas sociais em que estão imersos. Isso ocorre por meio de práticas sociais pelas quais acessam quadros de referência (estruturas) disponíveis e resultantes da atividade social (vide Emirbayer \& Mische, 1998 e Giddens, 2003). Diante disso, todos os elementos aqui avaliados para ilustrar a agência não são resultados exclusivos da ação individual, mas produto social, embora se admita, é claro, que a capacidade da agência é estratificada, assim como seu efeito sobre o campo. Deste modo, ao adotarmos uma perspectiva estruturacionista como orientadora deste estudo, consideramos a teleologia do agente não como fator explicativo para a configuração 
das relações e do significado relacional, mas apenas como explicação de seus propósitos no seu nível de reflexividade sobre o sistema social.

À guisa de conclusão, em face dos apontamentos aqui dispostos, apresentamos como sugestão de novas pesquisas a tentativa de aproximação teórica com os estudos ligados a movimentos sociais ou mais propriamente, movimentos intelectuais. Mecanismos sociais de socialização, mobilização e enquadramento podem auxiliar na compreensão do papel da agência no processo de produção do conhecimento científico e, em face de novas possibilidades que possam advir dessa consideração, recomendamos que a associação entre movimentos intelectuais e a construção do conhecimento científico constitua foco de futuras pesquisas.

\section{Referências}

Acedo, F., Barroso, C., Casanueva, C. \& Galán, J. (2006). Co-authorship in management and organizational studies: an empirical and network analysis. J ournal of Management Studies, 43(5), 957-983.

Alexander, J. C. (1984). Social-structural analysis: some notes on its history and prospects. The Sociological Quarterly, 25(1), 5-26.

Astley, W. G. \& Van De Ven, A. H. (1983). Central perspectives and debates in organization theory. Administrative Science Quarterly, 28(2), 245-273.

Barabasi, A. (2005). Network Theory - The Emergence of the Creative Enterprise. Science, 308(5722), 639-641.

Barabasi, A.; Albert, R. (1999). Emergence of Scaling in Random Networks, Science, 286(5439), 509-512.

Bardin, L. (1977). Análise de Conteúdo. Lisboa: Edições 70.

Battilana, J. (2006). Agency and Institutions: The Enabling Role of Individuals' Social Position. Organization, 13(5), 653-676.

Beaver, D. \& Rosen, R. (1979). Studies in Scientific Collaboration: Part III Professionalization and the Natural History of Modern Scientific Co-authorship. Scientometrics, 1(3), 231-245.

Bonacich, P. (1987). Power and Centrality: A Family of Measures. American J ournal of Sociology, 92(5), 1170-1182.

Bozeman, B. \& Corley, E. (2004). Scientists Collaboration Strategies: Implications for Scientific and Technical Human Capital. Research Policy, 33(4), 599-616.

Braun, T., Glänzel, W. \& Schubert, A. (2001). Publication and Cooperation Patterns of The Authors of Neuroscience Journals. Scientometrics, 51(3), 499-510.

Cohen, I. (1999). Teoria da estruturação e práxis social. In: Giddens, A. \& Turner, J. (Org.), Teoria Social Hoje. São Paulo: Unesp. p. 393-446.

Dimaggio, P. (1995). Comments on "What theory is not". Administrative Science Quarterly, 40(3), 391-397.

Emirbayer, M. \& Mische, A. (1998). What is Agency? American J ournal of Sociology, 103(4), 962-1023. 
Emirbayer, M. (1997). Manifesto for a Relational Sociology. American Journal of Sociology, 103(2), 281-317.

Fligstein, N. (1999). Fields, power, and social skill: a critical analysis of the new institutionalism. (Rep. No. Paper wps-199-01). Center for Culture, Organizations and Politics: Stanford.

Fligstein, N. (2001). Social skill and the theory of fields. Sociological Theory, 19(2), 105-1025.

Frickel, S. \& Gross, N. (2005). A general theory of scientific/intellectual movements. American Sociological Review, 70(2), 204-232.

Friedkin, N. E. (1998). A Structural Theory of Social Influence. Cambridge: Cambridge Univesity Press.

Glänzel, W. \& Schubert, A. (2008). Analysing Scientific Networks through CoAuthorship. In H. F. Moed, W. Glänzel \& U. Schmoch (Eds.), Handbook of Quantitative Science and Technology Research (pp. 257-276). New York: Kluwer Academic Publishers.

Giddens, A. (1978). Novas Regras do Método Sociológico. Rio de Janeiro: Zahar.

Giddens, A. (1989). A Constituição da Sociedade. São Paulo: Martins Fontes.

Giddens, A. (2001). Funcionalismo: après la lute. In: Giddens, A. (Org.). Em defesa da sociologia. São Paulo: Unesp. p. 115-159.

Giddens, A. (2003). A constituição da sociedade. (2 ed.). São Paulo: Martins Fontes.

Granovetter, M. S. (1985). Economic Action and Social Structure: The Problem of Embeddedness. American J ournal of Sociology, 91(3), 481-510.

Guarido Filho, E. R. (2008). A Construção da Teoria Institucional nos Estudos Organizacionais no Brasil: o período 1993-2007. Tese de Doutorado em Administração, Universidade Federal do Paraná. Curitiba: UFPR.

Guarido Filho, E. R., Machado-da-Silva, C. L. \& Gonçalves, S. A. (2009). Organizational Institutionalism in the Academic Field in Brazil: Social Dynamics and Networks. Brazilian Administration Review, 6(4), 299-315.

Guimera, R., Uzzi, B., Spiro, J. \& Amaral, L. A. N. (2005). Team Assembly Mechanisms Determine Collaboration Network Structure and Team Performance. Science, 308(5722), 697-702.

Hair, J., Anderson, R. E., Tatham, R. L. \& Black, W. C. (2005). Análise Multivariada de Dados. Porto Alegre: Bookman, 2005.

Hanneman, R. A., \& Riddle, M. (2005). Introduction to Social Network Methods. Riverside: University of Califórnia.

Katz, J. S. \& Martin, B. R. (1997). What is Research Collaboration? Research Policy, 26(1), 1-18.

Kuhn, T. S. (1978). A Estrutura das Revoluções Científicas. (2 ed.). São Paulo: Perspectiva.

Lee, S. \& Bozeman, B. (2005). The Impact of Research Collaboration on Scientific Productivity. Social Studies of Science, 35(5), 673-702.

Machado-Da-Silva, C. L., Fonseca, V. S. \& Crubellate, J. M. (2005). Estrutura, Agência e Interpretação: Elementos para uma Abordagem Recursiva do Processo de Institucionalização. Revista de Administração Contemporânea, 9(Edição Especial), 09-39. 
Machado-Da-Silva, C. L., Guarido Filho, E. R. \& Rossoni, L. (2006). Organizational Fields and the Structuration Perspective: Analytical Possibilities. Brazilian Administration Review, 3(2), 32-56.

Machado-Da-Silva, C. L. \& Rossoni, L. (2007). Persistência e mudança de temas na estruturação do campo científico da estratégia em organizações no Brasil. Revista de Administração Contemporânea, 11(4), 33-58.

Mead, G. H. (1934). The Philosophy of the Present. Chicago: University of Chicago Press.

Melin, G. (2000). Pragmatism and Self-Organization: Research Collaboration on the Individual Level. Research Policy, 29(1), 31-40.

Merton, R. K. (1996). On social structure and science. Chicago: Chicago University Press.

Moody, J. (2004). The Structure of a Social Science Collaboration Network: Disciplinary Cohesion from 1963 to 1999. American Sociological Review, 69(2),213238.

Newman, M. E. J. (2001). The structure of scientific collaboration networks. Proceedings of the National Academy of Sciences of the United States of America, 98(2), 404-409.

Reed, M. (2003). The agency/structure dilemma in organization theory: open doors and brick walls. In: Tsoukas, H. \& Knudsen, C. (Eds.) The Oxford handbook of organization theory. New York: Oxford University Press. p. 289-309.

Rodrigues, S. B. \& Carrieri, A. P. (2001). A Tradição Anglo-Saxônica nos Estudos Organizacionais Brasileiros, Revista de Administração Contemporânea, 5(Edição Especial), 81-102.

Rossoni, L. \& Guarido Filho, E. R. (2009). Cooperação entre Programas de PósGraduação em Administração no Brasil: Evidências Estruturais em Quatro Áreas Temáticas. Revista de Administração Contemporânea, 13(3), 366-390.

Rossoni, L. \& Machado-Da-Silva, C. L. (2008). Relações entre Posições Sociais no Campo da Pesquisa em Estudos Organizacionais e Estratégia em Organizações no Brasil. In: Cruz, J. A. W., Martins, T. S., \& Augusto, P. O. M. Redes Sociais e Organizacionais em Administração. Curitiba: Juruá, 2008. p. 117-144.

Rousseau, B. \& Rousseau, R. (2000). Lotka: A program to fit a power law. International Journal of Scientometrics, Informetrics and Bibliometrics, 4(1), 1-6.

Scott, J. (2000). Social Network Analysis: a handbook. (2 ${ }^{\text {nd }}$. ed.) London: Sage Publications.

Scott, W. R. (2008). Lords of the Dance: Professional as Institutional Agents. Organization Studies, 29(2), 219-238.

Seo, M. G. \& Creed, W. E. D. (2002). Institutional Contradictions, Praxis, and Institutional Change: A Dialectical Perspective. Academy of Management Review, 27(2), 222-247.

Uzzi, B. \& Lancaster, R. (2004). Embeddedness and Price Formation in the Corporate Law Market. American Sociological Review, 69(3), 319-344.

Wagner, C. S. \& Leydesdorff, L. (2005). Network Structure, Self-Organization, and the Growth of International Collaboration in Science. Research Policy, 34(10), 1608-1618.

Wasserman, S. \& Faust, K. (1994). Social Network Analysis: Methods and Applications. Cambridge: Cambridge University Press. 
Weaver, G. R. \& Gioia, D. A. (1994). Paradigms lost: Incommensurability vs. structurationist inquiry. Organization Studies, 15(4), 565-590.

White, D. R., Owen-Smith, J., Moody, J. \& Powell, W. W. (2004). Networks, Fields and Organizations: Micro-Dynamics, Scale and Cohesive Embeddings. Computational \& Mathematical Organization Theory, 10(1), 95-117.

Wuchty, S., Jones, B. F. \& Uzzi, B. (2007). The Increasing Dominance of Teams in Production of Knowledge. Science, 316(5827), 1036-1039.

Zucker, L. G. \& Darby, M. R. (1996). Star scientists and institutional transformation: patterns of invention and innovation in the formation of the biotechnology industry. Proceedings of the National Academy of Sciences of the United States of America, 93(23), 12709-12719. 